VARIA

\title{
LA PROXIMITÉ DANS L'ESPRIT DU TEMPS
}

\author{
Caroline Huynen ${ }^{1}$
}

Le terme «proximité» envahit actuellement le vocabulaire aussi bien scientifique que journalistique. Beaucoup l'évoquent pour parler tout autant des nouvelles technologies de télécommunication, d'un phénomène général de société, d'un remède contre la crise de l'emploi voire même de certains médias, ou encore d'une arme politique pour remporter des élections. Michel Maffesoli n'hésite pas à associer ces multiples effets de proximité à une forme d'esprit du temps : “(...) à partir de constatations empiriques, de petites monographies ou d'observations journalistiques, on peut s'accorder sur la naissance d'un nouvel «esprit du temps». Prenant acte également d'une certaine faillite des grands systèmes explicatifs qui ont régi la modernité, on peut proposer une autre logique de l'être-ensemble. Logique qui ne serait plus finalisée, tournée vers le lointain, mais au contraire centrée sur le quotidien"2. Même les sciences humaines s'intéressent au proche dans le mode d'appréhension de leur objet. Marc Augé souligne le développement d'une «anthropologie du proche» qu'il décrit comme se détachant de l'étude des civilisations

1 Chercheur au Département de communication de l'Université catholique de Louvain.

2 M. MAFFEsoli, Au creux des apparences. Pour une éthique de l'esthétique, Paris, Plon, 1990, p. 42-43.

Recherches en communication, $\mathrm{n}^{\circ} 7,(1997)$. 
lointaines pour se recentrer sur l'Europe. Quant à Maffesoli, il propose de pratiquer une «sociologie de la caresse». Cette sociologie, selon lui typiquement postmoderne, met en œuvre une pensée d'accompagnement par opposition à la manière de penser plus surplombante de la modernité, qu'il associe à l'idée de griffure conceptuelle.

Nous voudrions circonscrire en deux temps ce phénomène de proximité multiforme qui touche de nombreux secteurs de la société et qui alimente aussi plusieurs discours scientifiques contemporains. Le premier temps est une interrogation sur l'aspect purement définitoire de la proximité ; le deuxième concerne la présence manifestement transdisciplinaire du terme proximité dans le discours tenu par quelques auteurs de sciences humaines. Parmi celles-ci, la sociologie est évidemment la mieux placée pour mettre au jour et interpréter cet esprit du temps puisqu'elle étudie directement les faits sociaux. Mais il est frappant de noter que la théorie de la littérature, la philosophie, la communication ou l'anthropologie évoquent elles aussi des mécanismes de proximité à l'œuvre dans de multiples domaines.

\section{Une proximité tripartite}

Le sens commun envisage habituellement trois types de proximité : d'abord une proximité géographique définie comme étant la "situation d'une chose qui est à peu de distance d'une autre, de plusieurs choses qui sont proches"; ensuite une proximité affective définie comme étant le "caractère de ce qui est proche (c'est-à-dire des liens de parenté)"; enfin une proximité temporelle définie comme étant le "caractère de ce qui est rapproché dans le temps passé ou futur". L'aspect évolutif de la définition qui passe progressivement à un degré figuré (de la "situation" au "caractère") est donc manifeste. Deux idées viennent à l'esprit pour compléter cette définition tripartite et éclaircir la question de la nature même de la proximité. Tout d'abord l'idée d' «entre-deux» apparaît inhérente à la proximité. Celleci peut en effet être perçue comme s'activant dans un espace intermédiaire, dans un intervalle entre deux ou plusieurs éléments. Elle suppose alors une prise de distance, un écart minimal entre au moins deux objets ou deux personnes. Elle n'implique pas une confusion totale, un effet de miroir entre deux ou plusieurs éléments parfaitement identiques. Cette idée d'entre-deux est notamment abordée par 
Jean Baudrillard et Marc Guillaume qui notent une mise à distance minimale nécessaire à l'acte de communication entre deux personnes. Ils écrivent : "(..) si tout est commun entre deux êtres, il n'y a plus de communication, elle s'évanouit dans une intimité trop grande. Il y a là une aporie classique : ce que vise la communication est aussi ce qui la fait disparaître. Autrement dit, toute communication repose sur ce qui lui est contraire et sur la séparation des êtres"1.

La deuxième idée qui découle de celle d' «entre-deux» repose sur le fait que dans l'espace intermédiaire qu'elle occupe, la proximité, loin d'être statique, opère un mouvement de va-et-vient entre des couples de termes contraires. Autrement dit, nous complétons la définition tripartite par ceci : la proximité géographique gère une tension entre les termes dichotomiques "proche $\leftrightarrow \rightarrow>$ lointain", la proximité affective gère une tension entre les termes dichotomiques "familier <-> étranger" et la proximité temporelle gère une tension entre les termes dichotomiques "imminent $<->$ éloigné". Bref, la proximité n'est pas un état fixe mais plutôt une position intermédiaire où s'effectue un mouvement de réduction des distances entre des couples de termes dichotomiques. Le raisonnement peut être poussé plus loin. En reprenant d'une part les trois couples dichotomiques de base que nous venons d'extrapoler à savoir proche versus lointain, familier versus étranger, imminent versus éloigné, et en nous référant d'autre part à la définition de l'adjectif "fluctuant" ("qui varie, va d'un objet à un autre et revient au premier"), nous posons l'hypothèse que la proximité prend en compte les termes contraires "lointainétranger-éloigné". Autrement dit elle opère nécessairement par fluctuation c'est-à-dire par "variations successives en sens contraire" et fait donc appel aussi bien au lointain, à l'étranger et à l'éloigné qu'au proche, au familier et à l'imminent. Elle suppose toujours une minimisation de la mise à distance, de l'écart ou de la différence. Bref nous intégrons les composantes de distance, de mouvement et d'opposition au sein même de la définition de la proximité.

Au terme de ce premier temps relatif à l'aspect purement définitoire de la proximité, avançons à titre récapitulatif la piste de réflexion suivante : la proximité occupe une position d'entre-deux et dans cet espace intermédiaire, elle n'est pas statique mais opère un mouvement de fluctuation et de réduction des écarts entre des termes dichoto-

1 J. Baudrillard, M. Guillaume, Figures de l'altérité, Paris, Descartes \& Cie, 1994, p. 26-27. 
miques. Autrement dit, nous nuançons la définition du sens commun : si celui-ci définit la proximité de manière statique (d'abord comme une «situation», ensuite au sens figuré comme un «caractère»), nous préférons plutôt l'envisager comme une sorte de «dynamique oscillatoire». Elle se définit ainsi comme une dynamique spatiale, affective ou temporelle qui suggère un mouvement intermédiaire de fluctuation et de va-et-vient, aussi réduit soit-il, entre des termes opposés (proche versus lointain ; familier versus étranger ; imminent versus éloigné). Autrement dit la proximité n'est pas un état fixe mais elle s'inscrit dans un mouvement réducteur des distances entre des termes opposés qui ne peuvent se confondre ou être absorbés l'un par l'autre.

Signalons que la manière dont notre approche définitoire opère une scission nette entre les sphères du proche et du lointain relève sans doute d'un héritage culturel. Les sociétés dont nous faisons partie et qui sont issues des cultures méditerranéennes ont en effet l'habitude de distinguer clairement le privé et le public ainsi que de manière plus générale le proche et le lointain, l'intérieur et l'extérieur. Par ailleurs d'autres questions peuvent naître de la prise en compte de notre définition dans laquelle nous introduisons la composante de la distance. En effet comment évaluer ce qui est proche et ce qui est lointain ? Comment en d'autres termes estimer la proximité et la distance? Les recherches de Bateson, Goffman, Hall ou Watzlawick ont tenté de répondre à ces questions. En effet ces auteurs introduisent le terme de «proxémique» tournant autour des notions de distance et de promiscuité qui varient d'une culture à l'autre. Ils pensent qu'il n'existe pas chez l'homme de mécanisme fixe d'appréciation des distances qui soit universel et valable pour toutes les cultures. Un des problèmes méthodologiques révélé par la recherche proxémique est d'ailleurs le suivant : d'abord les sujets sont incapables de décrire comment ils établissent leurs distances ; ensuite les divers groupes ethniques établissent leurs distances différemment. Les psychosociologues montrent bien que l'impression de proximité ou de distance se fonde sur des éléments comme le ton de la voix, l'attitude ou la distance de l'interlocuteur. Dans son livre intitulé La dimension cachée ${ }^{1}$ et à partir d'observations faites sur des individus placés dans un contexte social, Edward Hall établit quatre types de distance : intime, personnelle, sociale et publique. Toujours dans la perspective de mouvement oscillatoire qu'implique selon nous la proximité entre

1 E. Hall, La dimension cachée, Paris, Éd. du Seuil, 1971. 
ce qui est proche et ce qui est lointain, il est intéressant de noter qu'à chacune des quatre distances décrites, Hall adjoint deux modalités : le proche et le lointain.

\section{La proximité dans le discours des sciences humaines}

A partir de recherches actuelles ou plus anciennes réalisées par des auteurs de sciences humaines, les lignes qui suivent introduisent une série de concepts et de théories touchant au thème de la proximité. $\mathrm{Au}$ préalable nous tenons à insister sur deux points. Tout d'abord, l'objectif poursuivi ici n'est pas d'offrir un relevé exhaustif des sciences humaines qui ont inclus l'étude de la proximité dans leurs recherches. Il s'agit plutôt de dégager quelques travaux significatifs qui nous paraissent intéressants à confronter avec notre approche définitoire de la proximité. Ensuite l'abondance d'usage soit de la terminologie de la proximité soit de terminologies voisines rencontrées lors de notre tour d'horizon rapide des sciences humaines, témoigne du caractère visiblement riche et transdisciplinaire du terme proximité. Cette abondance révèle aussi toute la difficulté de proposer une définition unifiée de la proximité. C'est pourquoi nous choisissons comme fil conducteur de notre parcours exploratoire de quelques auteurs de sciences humaines, l'idée de dynamique oscillatoire entre des couples de termes opposés. Ainsi nous verrons comment chaque auteur retenu se situe par rapport à notre hypothèse de dynamique oscillatoire entre des termes opposés. Notons que dans notre définition générale de la proximité, le couple générique de termes opposés est celui formé par l'opposition entre le proche et le lointain. Certains auteurs que nous allons aborder dans les pages qui suivent, déclinent une série de couples de termes opposés qui tout en étant pas identiques sont selon nous corrélatifs à celui de «proche-lointain» à savoir : «même-autre», «privé-public», «intérieur-extérieur», «banalétrange».

Commençons tout d'abord par les auteurs dont les travaux rendent manifestement compte de la dynamique oscillatoire (telle que nous la proposons dans notre essai définitoire) entre le couple de termes opposés "proche-lointain" et par extension "même-autre". 


\section{Le proche et le lointain}

Le sociologue allemand Georg Simmel, dans un ouvrage intitulé Digression sur l'Étranger et publié en 1905, note un penchant naturel chez l'homme à prendre comme hypostase de l'autre, un autrui lointain, par exemple l'étranger ou le marginal. Il défend l'idée que l'étranger est à la fois celui qui est proche et loin. Loin ne signifie pas nécessairement qu'il y a une distance géographique ou culturelle mais plutôt qu'il y a un passage de frontière. Chez Simmel, le statut de l'étranger est celui de quelqu'un qui n'est pas juge et partie, qui n'est pas partial dans les débats et les conflits, qui ne reste pas, d'une façon permanente, engagé dans notre voisinage social. Il peut par conséquent être juge impartial, prendre une distance permettant de mieux observer les conflits et les situations qui ne le concernent pas durablement. Cette distance lui permet paradoxalement une proximité en lui conférant une position d'arbitre ou de confesseur car on se confie plus volontiers à l'étranger qu'à ses proches. La théorie de Simmel met donc en évidence que dans le rapport à l'autre interviennent deux dimensions, être à la fois loin et près. L'étranger est celui qui selon un certain axe est très loin et, selon un autre axe, très près. La réflexion de Simmel conforte donc notre idée que la proximité n'est pas un état fixe exclusivement confiné dans le domaine du proche ou de l'immédiat mais peut être conçue comme une situation intermédiaire qui, loin d'être statique, fluctue entre des dimensions proches et lointaines.

Les dialectiques «même-autre», «proche-lointain» sous-jacentes dans la théorie de Simmel, sont par ailleurs développées par le philosophe Paul Ricœur dans son ouvrage intitulé Soi-même comme un autre ${ }^{1}$. Ricœur note lui aussi une nécessaire distanciation minimale avec soi-même. Sa réflexion va dans le même sens que celle de Baudrillard et de Guillaume évoquée plus haut et relative à la distanciation minimale dans l'acte de communication. En effet Ricœur n'envisage le soi-même qu'avec un degré minime, «intime» écrit-il, d'altérité. Il existe en fait pour lui une véritable dialectique «identitéaltérité». L'identité c'est-à-dire la reconnaissance d'un soi, n'est possible que par la reconnaissance de l'autre. Autrement dit la prise en compte de l'altérité et de la différence est essentielle à la formation de l'identité et du soi.

1 P. RICGUR, Soi-même comme un autre, Paris, Éd. du Seuil, 1990. 
Illustrons cette idée de nécessaire structuration du sujet par l'autre en prenant un cas bien précis d'appropriation de l'autre mis en place par les individus dans la société actuelle. Il s'agit de la communication par projection dans les idoles. Les mass media offrent souvent à l'individu la possibilité de se projeter c'est-à-dire de déléguer aux idoles de la télévision, du cinéma, des magazines illustrés, la vie dont il rêve. Il s'agit pour lui d'améliorer son existence souvent morne et banale en se réappropriant en rêve la vie d'autres hommes auréolés de gloire, de richesse, de beauté ou de célébrité. Ces «autres» parfois divinisés deviennent malgré tout familiers, intimes si l'on prend en compte l'abondance de détails sur leur vie privée offerts dans les médias. La familiarité est parfois tellement extrême que nous sommes plus éloignés de nous-mêmes que de ces «autres» idéalisés :

Là est le paradoxe : aventuriers cathodiques, surfers cybernétiques, nous semblons oublier que nous ne voyageons et ne communiquons plus que par procuration. Et que c'est la vie des autres qui défile dans notre salon, pas la nôtre. Que s'est-il passé ? Comment, militant pour une vie de grands espaces et de grands horizons (grande aventure, grand amour, grandes idées), en sommes-nous si souvent réduits à nous comporter en retraités de l'existence, ne pratiquant plus «la belle vie» que dans un fauteuil où nous convoquons, programmons des émotions non assouvies ?

La définition de la proximité que nous envisageons sous l'aspect d'une dynamique oscillatoire entre des couples de termes opposés et comme intégrant la composante de la distance est indirectement remise en cause par une série d'auteurs contemporains qui réfléchissent la société non pas en terme de proximité telle que nous l'avons définie mais sous la forme de repli sur le proche ou encore de confusion accrue des termes «proche» et «lointain» (normalement clairement opposés et distincts dans notre définition de départ). Dans ces deux cas la dynamique oscillatoire et la distance disparaissent soit au profit de situations plus inertes où la société baigne véritablement dans le proche sans aucune mise à distance, soit au profit d'états fusionnels où l'on ne parvient plus à distinguer le proche du lointain. Les auteurs que nous allons aborder dans les pages qui suivent témoi1 N. CROUSSE, "Les idoles peuvent-elles guérir l'ennui ?", Le Vif/L'Express, 8-15
février 1996, p. 39. 
gnent d'abord de ce mouvement de repli sur le proche, ensuite de ce brouillage entre les sphères distinctes du proche et du lointain.

\section{Repli sur le proche}

L'historien des mentalités Georges Balandier témoigne de la percée des sciences humaines dont les objets de recherche refluent sur les espaces et les temporalités du quotidien et qui accordent aujourd'hui une importance toute particulière à l'étude d'un esprit du temps centré sur le proche et l'ordinaire :

Le plus important (peut-être) dans la vogue qui multiplie les recherches portant sur la quotidienneté est le mouvement récent des esprits qui a fait reparaître le sujet face à la quantité, le vécu face à l'institué. Cette tendance forte affecte bien plus que le seul champ des sciences sociales, mais l'affecte principalement. De ce point de vue, il n'est pas sans intérêt de constater que la sociologie du quotidien (...) rejoint avec un certain succès deux des disciplines célébrées durant les vingt dernières années, l'anthropologie sociale, culturelle, historique (qui considère le rapport à l' «autre») et la psychanalyse (qui traite de la relation de l'individu à sa propre histoire). Dans les trois cas, le point de vue du sujet est privilégié sans qu'il s'agisse nécessairement d'un sujet relevant de l'exceptionnel, mais plutôt de 1'"ordinaire" ou du "banal".

On peut s'interroger sur les origines de ce retour au quotidien, de ce repli sur le proche et trouver des éléments de réponse dans le champ de la théorie de la littérature. Ainsi le livre de Daniel Madelénat intitulé L'intimisme ${ }^{2}$ met au jour une esthétique intimiste dans la littérature depuis la moitié du dix-huitième siècle jusqu'à l'aube du vingtième siècle. L'auteur fait ressortir les liens de l'artiste avec son microcosme ordinaire, son art d'apprivoiser, d'orner, de figurer l'humble et le quotidien. Pour Madelénat cet intimisme préfigure en fait la retraite contemporaine et typiquement postmoderne sur le moi et son environnement immédiat. Sa démarche est intéressante dans la mesure où elle remonte le temps pour trouver au cours des deux siècles précédant le vingtième siècle les germes de la recherche

1 G. Balandier, "Essai d'identification du quotidien" in Sociologie des quotidiennetés, Cahiers internationaux de sociologie, LXXIV, 1983, p. 7-8.

2 D. Madelénat, L'intimisme, Paris, PUF, 1989. 
d'intimité qui obsède l'homme postmoderne. C'est donc aussi une réflexion sur l'air du temps que Madelénat caractérise notamment par le couple «proximité et empathie» auquel il associe les expressions suivantes : histoires de vie, retour de la quotidienneté, sociologie qui se micronise, hyperindividualisme et narcissisme, rubriques de magazine intitulées "Aujourd'hui", "Les gens", "Notre temps"... Le repli sur le proche est d'ailleurs traduit par Madelénat dans la réflexion suivante :

Recru d'illusion, dépris d'utopie, saturé jusqu'à la nausée d'images violentes, l'homme postmoderne semble replier les ailes de ses songes sur l'espace intime d'une quotidiennetérefuge : foyer, famille, voisinage ; (...) [L'individu] s'enclôt dans le royaume des petits riens: loin du sublime ou de l'exception, il cultive l'ordinaire et le banal. L'hyperindividualisme et le narcissisme -tant de sociologues l'affirment- caractérisent l'esprit du temps'.

Pour illustrer ce que décrit Balandier à propos de la percée des sciences humaines qui se focalisent sur le proche, référons-nous par exemple à la sociologie du quotidien et à l'un de ses représentants : Michel Maffesoli. Celui-ci affirme que la proximité est typique de la postmodernité. Il parle d'une esthétique postmoderne dont la fonction plus agrégative que représentative trouve à s'exercer dans l'image, le mythe et l'allégorie qui nourrissent le réenchantement postmoderne. Alors que la modernité privilégie les individus et leurs associations contractuelles et rationnelles, Maffesoli pense que la postmodernité met l'accent sur la dimension affective et sensible. Si la modernité a été marquée par la politique, la postmodernité l'est par le clan. A ce sujet Maffesoli intègre la notion de «proxémie» qu'il caractérise par deux aspects : retour de l'importance du rôle joué par la communauté et intérêt manifeste pour les histoires vécues au jour le jour. A partir de cette notion de proxémie, il suggère même d'établir une sorte de loi sociologique dualiste qui repose sur le choix entre deux modes possibles de saisie du réel : le lointain ou le localisme. Cette loi sociologique s'organise autour de la prédominance accordée ou non à l'image. Ainsi,

Chaque fois que la méfiance vis-à-vis de l'image tend à prévaloir (ce sont les périodes d'iconoclasme, de monovalence

1 lbid., p. 11. 
rationaliste) s'élaborent des représentations théoriques et des modes d'organisation sociale qui ont le "lointain" comme dénominateur commun ; on assiste alors à la domination de la politique, du linéarisme historique, toutes choses qui sont essentiellement prospectives. Par contre quand l'image sous ses diverses modulations revient sur le devant de la scène, c'est alors le localisme qui devient une réalité incontournable ${ }^{1}$.

La fonction agrégative typiquement postmoderne dont parle Maffesoli est liée selon lui au retour d'une forme de proximité tribale. En effet les nouvelles tribus postmodernes qu'il décrit développent une communication tactile qui a comme seul objectif de «toucher» ou de «se toucher» c'est-à-dire de participer à une forme de grégarité et de non-différenciation entre les individus. Peu importe finalement le contenu du message véhiculé entre ces tribus. D'après Maffesoli cette communication ou plutôt cet être-ensemble concerne principalement les médias dans leur communication du sport, de la musique ou de la consommation. Ainsi dans la postmodernité, l'émotion ne peut pas être réduite à la seule sphère du privé, mais se vit, de plus en plus, collectivement. On peut même parler d'une ambiance affectuelle où les peines et les plaisirs sont éprouvés en commun. Il suffit de faire référence au rôle que joue la télévision lors de catastrophes, guerres ou autres événements sanglants, grandes commémorations nationales ou internationales, mariages princiers ou manifestations mondaines mettant en scène les stars de la chanson ou les vedettes de cinéma. Dans chacun de ces cas le média permet de vibrer en commun. C'est pourquoi Maffesoli pense que la postmodernité introduit un style communautaire qui rompt avec la logique de l'identité de la modernité, et donc avec le principe d'individuation au profit du principe de massification et de perte dans un sujet collectif. Ce processus de massification annule en quelque sorte la différence minimale entre deux personnes que décrivent Baudrillard et Guillaume dans l'acte de communication. Le regard théorique que porte Maffesoli sur la postmodernité témoigne bien de ce repli sur le proche décrit par Balandier et aussi d'une certaine forme d'inertie entrainée par l'annulation de toute dynamique oscillatoire entre le proche et le lointain (ces deux composantes étant d'ailleurs, dans la loi sociologique de Maffesoli, clairement exclusives ou alternatives et non pas opposées).

1 M. MAFFESOLI, Le temps des tribus. Le déclin de l'individualisme dans les sociétés de masse, Paris, Méridiens Klincksieck, 1988, p. 168. 
La perte de la distance minimale, exprimée chez Maffesoli dans la massification grégaire des tribus postmodernes ainsi que dans la communication tactile, s'écarte donc de l'idée de dynamique oscillatoire intégrée à notre définition de la proximité. En effet dans la théorie de Maffesoli, les individus ne se distinguent plus entre eux puisqu'ils se fondent dans une masse grégaire. L'altérité change de forme pour refluer vers le proche : "Le barbare n'est plus à nos portes, il est dans nos murs, il est en chacun de nous"l. Autrement dit l'altérité ne joue plus la différence et la distance mais elle devient proche et ressemblante. Maffesoli pense d'ailleurs qu'il faut actuellement tenir compte de cette présence structurale de l'autre au cœur du moi pour comprendre la socialité postmoderne.

L'on peut confronter au regard que porte Maffesoli sur la société celui sans doute plus pessimiste d'un autre sociologue du quotidien : Claude Javeau. Ce dernier n'évoque pas vraiment la proximité mais aborde les notions de banal et de quotidien. Javeau introduit l'expression de degré zéro du quotidien synonyme pour lui du banal. D'une part il pense que certaines institutions ont pour fonction d'imposer et de renforcer la banalité dans la vie quotidienne. Les mass media sont pour lui les véhicules privilégiés d'une banalité équivoque (celle d'une «ouverture» sur le vaste monde et d'une «fermeture» sur l'espace domestique). Parmi les symboles les plus explicites de cette banalité, Javeau place en premier lieu la télévision. D'autre part il écrit : "Le banal (...) est (...) le refuge assumé en tant que tel des individus qui pratiquent, dans leur vie de tous les jours, une continuelle dichotomie. Le banal (...) est à l'opposé de l'étrange (de l'aventureux, du romanesque, du bizarre). Le «degré zéro du quotidien» entend rompre avec un quotidien ludique, perpétuellement recréé" 2 . Pour Javeau le banal qui caractérise notre société est traversé par une forme d'inertie. Ce qui lui inspire les questions suivantes : l'attachement à la pesanteur du quotidien, le refus de bouger et d'atteindre l'étrange, ne manifestentils pas le refus d'être contrôlé dans les mouvements propres de la vie en société ? L'intensification du banal ne s'accompagne-t-elle pas parallèlement d'un désintérêt pour la vie en société ? L'attachement à la pesanteur du quotidien et du banal, cette sorte d'engluement et de refus de bouger, c'est-à-dire ce refus d'aller vers l'étrange, l'aventu-

1 M. MAFFESOLI, Éloge de la raison sensible, Paris, Grasset, 1996, p. 11.

2 C. JAVEAU, La société au jour le jour. Écrits sur la vie quotidienne, Bruxelles, De Boeck-Wesmael, 1991, p. 149. 
reux, le bizarre annulent ainsi le mouvement oscillatoire minimal entre des éléments distincts et opposés que nous envisageons comme étant un élément fondamental de la définition de la proximité.

En anthropologie, les travaux actuels de Marc Augé illustrent également le repli des sciences humaines sur le proche. Ils révèlent en effet un souci contemporain de micro-observations, de qualitatif, de témoignages directs. Augé parle de la nécessité de pratiquer une anthropologie du proche car "Tout ce qui éloigne de l'observation directe du terrain éloigne aussi de l'anthropologie (...)"'. Il montre que l'anthropologie actuelle a tendance à se replier sur l'Europe pour deux raisons : d'une part parce que les cultures exotiques n'apparaissent plus si différentes ou lointaines, d'autre part parce que le lointain ou l'autre devient géographiquement proche dans la société actuelle. "Aujourd'hui la planète a rétréci, l'information et les images circulent et, du même coup, la dimension mythique des autres s'efface. Les autres ne sont plus si différents (...). L'altérité demeure mais les prestiges de l'exotisme se sont évanouis. Le rapport à l'autre s'établit dans la proximité, réelle ou imaginaire. Et l'autre sans les prestiges de l'exotisme, c'est simplement l'étranger, souvent craint moins parce qu'il est différent que parce qu'il est trop proche"2. Nous pouvons donc mettre en relation cette idée avec la réflexion de Simmel à propos de l'étranger qui est selon un certain axe très loin et selon un autre axe très près.

Augé observe aussi une mutation des sociétés actuelles de moins en moins caractérisées par des prises de distance. A ce sujet il introduit la notion de surmodernité qu'il caractérise par la figure du triple excès : temporel, spatial et individuel. Tout d'abord au niveau temporel, Augé décrit une histoire difficilement pensable en raison de la surabondance événementielle produite notamment par les médias. L'histoire est constamment sur nos talons et n'offre plus la possibilité de la distance critique. Ensuite il relève un excès spatial paradoxalement corrélatif au rétrécissement de la planète. La surabondance spatiale s'exerce dans les changements d'échelle, dans la multiplication des références imagées et imaginaires ainsi que dans les spectaculaires accélérations des moyens de transport. Enfin au niveau individuel, Augé met au jour tout comme Balandier le retour de l'ego et de

1 M. AUGÉ, Non-lieux. Introduction à une anthropolgie de la sumodernité, Paris, Éd. du Seuil, 1992, p. 16-17.

2 M. AugÉ, Pour une anthropologie des mondes contemporains, Paris, Aubier, 1994, p. 26. 
l'individu, l'individualisation des références, la fluctuation des repères de l'identification collective, l'impuissance confirmée (aussi par d'autres auteurs comme Maffesoli, Vattimo ou Lyotard) des grands systèmes d'interprétation.

\section{Brouillage des frontières entre le proche et le lointain}

Abordons maintenant une série d'auteurs qui s'écartent de la définition de la proximité que nous donnions au début de cet article, en témoignant d'un brouillage entre les sphères (distinctes dans notre définition de départ) du proche et du lointain et par extension du privé et du public, de l'individuel et du collectif, de l'intérieur et de l'extérieur.

Ainsi plusieurs auteurs de sociologie politique parlent d'une confusion accrue entre la vie publique et la vie privée et même d'une intimité qui devient publique et qui s'impose aujourd'hui de manière tyrannique. A noter que ces deux aspects sont abordés de manière négative comme porteurs d'un danger pour la société.

S'agissant d'abord de la confusion accrue entre la vie publique et la vie privée, rappelons que les cultures méditerranéennes favorisent le cloisonnement entre le privé et le public tandis que la culture anglosaxonne préfère la transparence de la vie privée. Or les cultures méditerranéennes ont de plus en plus de mal à gérer la dichotomie anciennement établie entre privé et public. En effet, elles passent d'une société dirigée par les autres à une société dirigée de l'intérieur, avec toute la difficulté de savoir exactement par quoi est constitué cet intérieur. Il se produit donc une confusion, un rapprochement extrême entre la vie publique et la vie privée. Autrement dit les gens traitent en termes de sentiments personnels des affaires qui relèvent plutôt du public.

S'agissant ensuite du deuxième aspect important souligné par la sociologie politique, Richard Sennett, dans son livre Les tyrannies de l'intimité, remarque que jamais les gens ne se sont autant passionnés pour des histoires de vie, ils ont de plus en plus une vision intimiste de la société. Le monde extérieur (public, politique, etc.) leur semble tellement vide, morne, éloigné de leurs préoccupations quotidiennes qu'ils veulent le rendre plus proche en le remplissant par des artifices de chaleur, de confiance, etc. Sennett observe ainsi une politique qui se personnalise et se nourrit exclusivement de nouvelles valeurs telles 
que la cordialité, les confidences intimes, la proximité, l'authenticité, la personnalité. Pour prendre un exemple concret on se rappelle que toute la campagne présidentielle de Jacques Chirac a effectivement joué sur cette accentuation des relations de proximité et de cordialité. Toutefois Sennett met en garde contre la poussée d'une intimité tyrannique. Il écrit :

Dans la mesure où cette tyrannie triomphe, la société ellemême est déformée. Je ne prétends pas (...) que nous comprenions intellectuellement les institutions et les événements en les rapportant systématiquement à des personnalités (...). Mais nous ne nous intéressons à ces institutions ou à ces événements que lorsqu'ils sont liés à des personnalités. L'intimité est à la fois une vision des relations sociales et une exigence. Seul compte ici ce qui est proche ou immédiat. Plus cette tyrannie de la proximité s'impose, plus les gens cherchent à se libérer des coutumes, des manières sociales, des codes, etc., pour s'ouvrir de façon inconditionnelle les uns aux autres ${ }^{1}$.

Il n'y a pas que le monde politique qui soit caractérisé par un brouillage des frontières entre le proche et le lointain c'est-à-dire entre le privé et le public, l'intérieur et l'extérieur. En effet les médias sont manifestement marqués ces dernières années par des traitements à résonance intime voire voyeuristes de la réalité. Dans son livre intitulé La télévision de l'intime, Dominique Mehl montre que le petit écran est devenu un grand confessionnal public, un lieu où se met en scène aujourd'hui la compassion moderne. Des émissions télévisées françaises telles que Perdu de Vue (TF1), Pour la vie (TF1), Bas les masques (France 2) confirment l'attrait actuel du public pour la surexposition du sentiment et de l'émotion ainsi que pour la mise en scène publique de la vie privée et de ses fractures. L'auteur développe une réflexion intéressante à propos de l'intimité confirmant l'idée de confusion entre les sphères normalement distinctes du privé et du public, du proche et du lointain. Elle écrit :

«Vie privée publique», «Vie publique privée» : cette extraordinaire imbrication du personnel et du collectif, ce brouillage des frontières entre le dedans et le dehors suscitent, depuis un certain temps, un flot de discours alarmistes. Pour ceux qui s'indignent de telles exhibitions, les nouvelles mises en scène publiques seraient destructrices de l'intimité. (...) La vie

1 R. SENNETT, Les tyrannies de l'intimité, Paris, Éd. du Seuil, 1979, p. 274. 
privée, en sortant de ses murs domestiques ou personnels, en se livrant à l'observation, la louange ou la condamnation de la collectivité, aurait perdu sa propriété essentielle : son mystère et son quant-à-soi. L'idée même d'intimité serait réduite à néant ${ }^{1}$.

Mehl nuance toutefois ce type de raisonnement: pour elle la mise en scène publique de l'intimité ne tue pas l'intime mais le reformule et redélimite son cadre définitoire. En effet "La thématique de l'intimité a longtemps été (...) circonscrite aux événements géographiquement identifiables au noyau familial et à la sphère domestique d'un côté, aux réseaux électifs privés et à la sphère relationnelle de l'autre. L'intimité devient aujourd'hui une idée mouvante, dont les limites sont tracées par chaque individu et non plus par une autorité sociale, politique, juridique, religieuse ou morale"2.

La position qu'adopte Baudrillard sur le devenir de l'intimité est nettement plus catégorique que celle de Mehl. D'une part, les médias ne produisent finalement qu'une intimité spectacle ou simulacre. Baudrillard affirme : "On voit revenir une espèce de sentimentalité tous azimuts, une réactivation sentimentale des valeurs perdues, le néo-romantisme, la nouvelle affectivité, "la passion à l'ordre du jour". Tout est repris en main par les médias ; il ne s'agit plus d'intimité, d'amour, d'affectivité mais de leur modèle. Ce n'est pas l'original mais la version sous-titrée, modèle de simulation d'un univers publicitaire"'3. D'autre part, la mise en contact permanente assurée par les médias exclut selon Baudrillard toute intimité :

Ce qui menace l'intimité dans nos sociétés, ce n'est pas tant la promiscuité que la transparence ; ainsi on peut la définir négativement : c'est ce dont on est privé par la transparence des flux, la circulation indéfinie des choses, l'accélération des systèmes, la communication qui (...) exclut l'intimité. Il s'agit de toujours être branché, en contact ; or pour être dans sa propre intimité, il faut avoir cette possibilité d'être hors circuit, «débranché» 4 .

1 D. MEHL, La télévision de l'intime, Paris, Éd. du Seuil, coll. Essai politique, 1996, p. 155.

2 Ibid., p. 159.

3 J. BAUDRILLARD, "La sphère enchantée de l'intime", in Autrement n 81, juin 1986, p. 14-15.

4 Ibid., p. 14. 
Cet «hors circuit» et ce débranchement rendus pratiquement impossibles prouvent une fois de plus la difficulté actuelle de la société de se mettre à distance. Toujours à propos de l'idée de mise en contact permanente, l'expression de «révolution proxémique» liée aux pratiques sur réseaux internationaux comme Internet se répand de plus en plus. Ces réseaux autorisent la mise en relation de personnes qui sans eux ne communiqueraient probablement jamais parce qu'elles sont distantes de quelques frontières ou quelques océans. Il y a donc révolution proxémique dans la mesure où les distances géographiques sans être gommées ne constituent désormais plus l'obstacle majeur à la communication. Ce sentiment extraordinaire de contact (cette communication tactile dirait Maffesoli) se jouant des distances géographiques ne doit pas masquer un contenu qui doit délivrer malgré tout un projet et un intérêt commun. Dans son livre Figures de l'altérité, Baudrillard observe justement la perte progressive de valeurs communes et parle d'une prolifération de formes nouvelles d'expression et de communication de type spectral, permises à la fois par des dispositifs techniques et surtout par un changement des sensibilités sociales. Celui-ci reflète pour Baudrillard

(...) la fin des communautés traditionnelles, l'affaiblissement des institutions intermédiaires de socialisation, les risques d'anomie et de solitude engendrés par des potentialités sociotechniques comprenant la multiplication des réseaux de connexions urbaines et tous les dispositifs de communication à «géométrie variable». A cet égard, l'explosion de Mai 1968 (...) semble être le dernier soubresaut d'un régime de socialité définitivement révolu. (...) En 68, on descend dans la rue, on rejoint des groupes en fusion, c'est l'espoir d'une communauté peut-être éphémère mais festive, communielle (...). Alors qu'aujourd'hui, on est vraiment dans un monde de réseaux multiples qui permettent un essor de nouvelles formes de socialité, n'ayant rien à voir avec cet embrasement des groupes en fusion. (...) Il s'agit d'une nouvelle forme de communicabilité qui nous fait rompre avec la nostalgie de la communauté, avec la dialectique traditionnelle de l'individuel et du collectif. Ce nouveau mode d'être et d'échanger, [Baudrillard] le qualifie de spectral ${ }^{1}$.

Il semble donc que l'univers de la communication contribue fortement à opérer un brouillage des frontières entre le proche et le

1 J. BAUDRILARD, M. GUILLAUME, op. cit., p. 22-23. 
lointain, le privé et le public, l'individuel et le collectif, l'intérieur et l'extérieur. Ce brouillage remplace dans certains cas l'idée de proximité (comme dynamique oscillatoire et comme distanciation minimale entre les sphères génériques bien distinctes du proche et du lointain) par celle de simultanéité. Paul Virilio affirme d'ailleurs à propos de la temporalité des médias: "La dimension temporelle du journalisme est une simultanéité nivelante. Tout a plus ou moins la même importance. Tout n'est que quotidien"'. A l'idée de simultanéité, l'on peut aussi associer celle de contemporanéité avancée par Gianni Vattimo. Pour celui-ci le monde actuel "n'est pas contemporain en raison de critères banals de proximité "chronologique" (contemporain au sens de ce qui est le plus proche de nous dans le temps), mais plutôt en tant que monde où se dessine et où commence à se réaliser la tendance à la réduction de l'histoire au plan de la simultanéité, au moyen de techniques comme celle de l'information télévisée en direct"2. Autrement dit il n'est plus toujours évident aujourd'hui de se construire des repères temporels qui permettent d'introduire la distance par rapport à un quelconque passé, présent ou futur. Les notions de simultanéité et de contemporanéité sont ainsi à l'origine de cette utopie d'une démocratie directe engendrée par les médias et dénoncée notamment par Olivier Mongin :

Une indistinction croissante du réel et de la fiction s'accompagne d'un brouillage des frontières du privé et du public. Mais elle en est aussi le ressort idéologique : l'utopie de la démocratie directe féconde en effet l'idéologie de la communication. Une idéologie d'autant plus invisible qu'elle se nourrit justement de l'illusion de maîtriser le temps et l'espace : «la communication, écrit Dominique Wolton, a triomphé dans les quarante dernières années en vainquant définitivement les deux obstacles qui depuis toujours la contraignaient : le temps et l'espace. Si elle veut conserver cette dimension émancipatrice, elle devra réexaminer les deux, le temps et l'espace, c'est-à-dire accepter de "perdre du temps" ce que déteste l'Occident» ${ }^{3}$.

1 P. VIRILIO (entretien), "Einstein l'avait annoncée. La bombe du troisième type", Globe hebdo, 27 octobre 1993.

2 G. Vattimo, La société transparente, Paris, Desclée de Brouwer, 1990, p. 29.

3 O. MONGIN, Face au scepticisme. Les mutations du paysage intellectuel ou l'invention de l'intellectuel démocratique, Paris, La Découverte, 1994, p. 25-26. 


\section{Conclusion}

S'il reste encore bien sûr à mesurer les effets et l'ampleur de telles formes nouvelles de communication, nous concluons notre réflexion par l'idée suivante : le terme proximité qui envahit le vocabulaire contemporain pour qualifier plusieurs situations ou comportements sociaux, n'est sans doute pas le plus approprié dans la mesure où la distance minimale et l'oscillation entre des termes clairement opposés que suppose selon nous la proximité, disparaissent progressivement. Peut-être faut-il plutôt parler de repli sur le proche ou de fusion dans le proche. En effet il ressort de quelques discours scientifiques contemporains que la société modifie son rapport au couple générique de termes opposés «proche-lointain». Elle a tendance soit à réduire de nombreux secteurs ou composantes (y compris le lointain, l'étranger, l'altérité) au proche, soit à fusionner le proche et le lointain au point qu'on ne parvient plus vraiment à distinguer les deux sphères. Il apparaît donc une certaine tendance de la société actuelle à s'écarter de toute une tradition héritée en partie des cultures méditerranéennes et qui l'a habituée à pratiquer souvent des scissions et des oppositions (qui n'excluent pas des dynamiques oscillatoires) entre le proche et le lointain, le privé et le public, le même et l'autre, le banal et l'étrange, l'intérieur et l'extérieur. A tel point que se manifeste parfois dans plusieurs discours scientifiques une nostalgie de la distance, de la coupure nette et de la perte du temps. 